\title{
ATIVIDADE FÍSICA E SINTOMAS DEPRESSIVOS EM NONAGENÁRIOS E CENTENÁRIOS ANTES E DURANTE O ISOLAMENTO SOCIAL
}

Ana Paula Tiecker; PUCRS; anapaulatiecker@hotmail.com Francielle Bonett Aguirre; PUCRS; franciellebaguirre@gmail.com Júlia de Freitas Machado; PUCRS; juliadfmachado@hotmail.com

Ângelo José Gonçalves Bós; PUCRS; angelo.bos@pucrs.br - Escola de Medicina da PUCRS, Porto Alegre, Brasil; - Projeto de extensão Atenção Multiprofissional ao Longevo (AMPAL) da PUCRS, Porto Alegre, Brasil

\section{RESUMO}

Introdução: A atividade física está relacionada à manutenção do bem estar físico e psicológico, cuja relação é pouco estudada em nonagenários e centenários. A Covid19 provocou um isolamento social que pode estar relacionado ao aumento de sintomas depressivos e diminuição da atividade física principalmente em nonagenários e centenários. Objetivo: Observar as possíveis mudanças na atividade física e sintomas depressivos durante a COVID19 em nonagenários e centenários. Metodologia: Estudo transversal e analítico com nonagenários e centenários participantes do projeto Atenção Multiprofissional ao Longevo (AMPAL) avaliados por telefone usando um instrumento multidimensional, que incluiu a Escala de Depressão Geriátrica (GDS) e a prática de atividade física, com o objetivo de verificar possíveis mudanças no comportamento antes e depois de 17/03/2020 (primeiro decreto municipal relacionado ao COVID19). As entrevistas foram feitas entre abril e agosto/2020. Resultados: Participa-

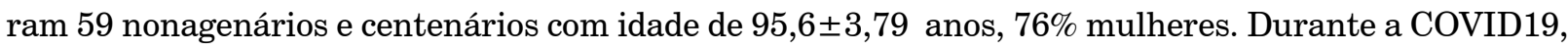
$39 \%$ dos participantes diminuíram a prática de atividade física e 15,2\% apresentou piora do GDS. Os participantes que responderam não praticar atividade física $51 \%$ apresentou nível de GDS indicativo de depressão. Conclusão: Observamos que a diminuição da prática de atividade física foi mais frequente do que a piora dos sintomas depressivos. Concluímos que a COVID19 está causando uma diminuição na prática da atividade física de forma mais precoce em nonagenários e centenários. Parece haver uma latência entre a diminuição da atividade física e o aumento dos sintomas depressivos a ser confirmada.

Palavras chaves: Saúde Publica; Envelhecimento; Pandemia.

Agradecimentos: "Este estudo foi parcialmente financiado pela Coordenação de Aperfeiçoamento de Pessoal de Nível Superior - Brasil (CAPES) - Código Financeiro 001”. 